\title{
A model comparison of flow and lateral sediment trapping in estuaries
}

\author{
G.P. Schramkowski \\ Flanders Hydraulic Research Laboratory, Antwerp, Belgium
}

K.M.H. Huijts \& H.E. de Swart

Institute for Marine and Atmospheric research Utrecht, Utrecht University, The Netherlands

H.M. Schuttelaars

Delft Institute of Applied Mathematics, Delft University of Technology, The Netherlands

Two different models for the distribution of flow and sediment over the cross-section of a tidally dominated channel are compared. The first is a state-of-the-art numerical model that solves the three-dimensional shallow water equations with prognostic density field. The second is an idealized model which includes residual and semi-diurnal tidal motions and uses a diagnostic residual density gradient as baroclinic forcing. For both models, an off-line sediment module is used to compute the lateral mean sediment distribution.

For fairly high values of vertical diffusivity $\left(\sim 0.01 \mathrm{~m}^{2} \mathrm{~s}^{-1}\right)$, a good qualitative agreement is found for residual flow patterns. The agreement of the amplitude of the semi-diurnal velocity components is satisfactory as well, although the phase distributions show deviations. The lateral mean sediment distributions are rather similar, and stem from a balance that is predominantly governed by mean concentration and residual currents. The flow patterns only differ qualitatively for either very low or very high tidal velocities. The sediment distributions only deviate for low tidal flow regimes.

\section{INTRODUCTION}

Observations in several estuaries show high sediment concentrations which are trapped near a particular bank (e.g. Nichols (1972), Geyer et al. (1998) and Fugate et al. (2007)). Examining lateral trapping of sediments is of considerable ecological and economical importance. For instance, the presence of high sediment concentrations will have a negative impact on biological activity. Also, the presence of high concentration of fine sediments increases the siltation rate of harbors.

Several modelling techniques are available to study lateral trapping of sediment. Complex numerical models provide detailed descriptions of estuarine flow and sediment dynamics. However, high computational costs and complexity make them less suited to gain physical insight. Simple idealized models provide analytical solutions which can be used to isolate physical mechanisms and to examine their effects on lateral trapping of sediment in a systematic way. However, simplifications and assumptions used to derive the analytical solutions may limit their applicability to real estuarine cross-sections. In this research project, a numerical and an idealized modelling approach are combined to profit from advantages of both modelling techniques. The numerical flow model that is used is TRIWAQ (e.g. Stelling (1984)). The idealized flow model is obtained from Huijts et al. (2007). For both models, the implications for lateral sediment trapping are investigated using the sediment module presented in Huijts et al. (2006).

The aim of this work is to gain physical understanding of the transverse distribution of flow and sediment in estuaries. In particular, this paper examines estuarine flow and sediment trapping for a range of tidal conditions. For each tidal condition, flows obtained with the numerical model and idealized model will be compared. Agreement in a particular range of tidal conditions will confirm that both flow models capture the main physics that is important for the flow in that range. The flow and sediment trapping mechanisms for those tidal conditions will be studied using the idealized model. For the remaining tidal conditions, disagreement will identify tidal conditions to which the idealized model is not applicable. For those conditions, the numerical model and sediment mod- 
ule will be used to study the estuarine flow and sediment trapping. Although the idealized model is not directly applicable to those tidal conditions, it might still be useful to interpret the physical processes that are important to the numerical flow and corresponding sediment distributions. This paper is organized as follows. In Sect. 2, the two hydrodynamic models and the sediment module are briefly outlined. Section 3 discusses the methodology that was used to compare the flow models. The results regarding flow and mean sediment distribution are presented in Sect. 4 and discussed in Sect. 5. In Sect. 6, finally, conclusions are drawn and future activities are outlined.

\section{FLOW AND SEDIMENT MODEL}

\subsection{Flow models}

\subsubsection{Idealized model}

Huijts et al. (2006) and Huijts et al. (2007) have developed a $2 \mathrm{DV}$ idealized model describing tidal and residual flow in a cross-section of weakly nonlinear, tidally-dominated estuaries. Tidal velocities are assumed to be an order of magnitude larger than residual velocities. The cross-section has an arbitrary bed profile. The model assumes along-channel uniform conditions and adopts a rigid lid approximation. The flow is described by the three-dimensional shallow water equations. Tides are forced by prescribing a semi-diurnal tidal discharge. Baroclinic effects are included by prescribing residual horizontal density gradients which are independent of height above the bed. The water surface is stress-free while a no-slip condition is imposed at the bed. Perturbation methods are used to obtain analytical solutions. At dominant order, the flow is a barotropic tide. At higher order, residual flow results from several effects, including horizontal density gradients, tidal advection of along-channel momentum and Coriolis forcing.

\subsubsection{Numerical model}

The numerical model is TRIWAQ, which solves the three-dimensional shallow water equations using a finite difference method. In contrast to the idealized model, TRIWAQ uses a free surface and resolves a salt balance equation. Also, Triwaq is able to compute flows in complex geometries and arbitrary bathymetry. In the horizontal direction, the domain is discretized using an orthogonal grid. Sigma-layers are used in the vertical direction. At the seaward side, a vertical $M_{2}$ tide (water level $\zeta$ ) is imposed. There is no water flux through the landward opening. The water surface is stress free and obeys a no-slip condition at the bed. Salinity is prescribed at the landward and seaward boundary, and a horizontal eddy diffusivity coefficient is used that varies arbitrary along-channel.

\subsection{Sediment module}

The sediment module is based on Huijts et al. (2006). The residual, semi-diurnal and quarterly diurnal components of the suspended sediment concentration are resolved from a sediment mass balance equation. The erosional flux of sediment at the bed is determined by the local bed shear stress and availability of bed sediment. The across-channel distribution of bed sediment is determined using a morphodynamic equilibrium condition

$$
F_{\mathrm{sed}}=\left\langle\int_{-\mathrm{H}}^{\zeta}\left[v c-K_{\mathrm{h}}^{\mathrm{sed}} \frac{\partial c}{\partial y}\right] d z\right\rangle=0,
$$

where $v$ is the across-channel velocity component, $c$ is concentration, $K_{\mathrm{h}}^{\text {sed }}$ is a horizontal diffusion coefficient for the sediment and $\langle$. $\rangle$ denotes averaging over a semi-diurnal tidal period. Hence, bed sediment is distributed across-channel such that the advective sediment transport balances the diffusive sediment transport if averaged over a tidal period. Note that the advective transport involves advection of the residual sediment concentration by the residual flow as well as net advective transport resulting from interaction between semi-diurnal and quarterly diurnal tidal constituents of the flow and sediment concentration. We take $K_{\mathrm{h}}^{\text {sed }}=5 \mathrm{~m}^{2} \mathrm{~s}^{-1}$. The sediment is noncohesive with a settling velocity of $0.3 \mathrm{~mm} \mathrm{~s}^{-1}$, which represents silty-clay material.

There are two differences in computing sediment concentrations for the idealized and numerical model flow. First, the quarterly diurnal flow is resolved in TRIWAQ, but not in the idealized model. Therefore, advection of the $M_{4}$-sediment concentration by the $M_{4}$ across-channel TRIWAQ flow induces additional lateral sediment transport which is not incorporated in the idealized model. Second, the harmonic constituents of the absolute value of the bed shear stress $(|\tau|)$ that are used as input in the sediment module are obtained in different ways. This quantity is obtained using the instantaneous TRIWAQ flow, but using only particular tidal constituents for the idealized model. The latter is a result of the assumption that the tidal flow is an order of magnitude larger than the residual flow. Differences in bed shear stress and hence sediment concentrations are expected if this assumption is violated.

\section{METHODS}

The numerical and idealized model are set up such that the geometric and physical conditions are similar in some part of the domain.

\section{$3.1 \quad$ Numerical model setup}

\subsubsection{Geometry and bathymetry}

The numerical model geometry consists of a straight tidal channel of length $L=1200 \mathrm{~km}$ and width $B=5$ 
$\mathrm{km}$ with vertical side walls (see Fig. 1). Flow and sed-

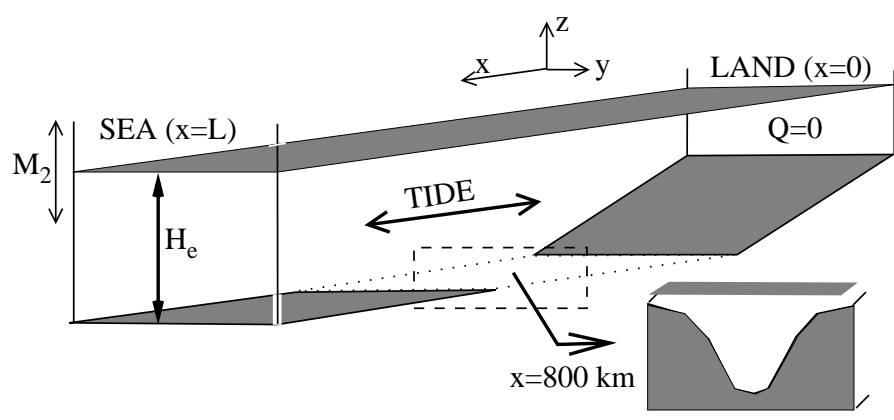

Figure 1: Schematic picture of the numerical model domain. The Gaussian cross-section at $x=800 \mathrm{~km}$ is shown enhanced. This slice is inside the intermediate region, which is indicated by the dotted lines. For further details, see main text.

iment dynamics are considered in a cross-section with a Gaussian bed profile at $x=800 \mathrm{~km}$ (see Fig. 1). Maximum and minimum water depth in this crosssection are $30 \mathrm{~m}$ and $2 \mathrm{~m}$, respectively. In a seaward region $(x>1100 \mathrm{~km})$, a horizontal bed is used to ensure that the incoming tidal wave is nearly linear and uniform in the cross-channel direction. To suppress reflection of the tidal wave at the landward boundary and enhance its damping, the landward region $(0<$ $x<500 \mathrm{~km}$ ) has a depth decreasing up-estuary from 12 to $3 \mathrm{~m}$. In an intermediate zone $(500<x<1100$ $\mathrm{km}$ ), the bathymetry changes gradually into the Gaussian cross-channel bed profile mentioned above.

\subsubsection{Salinity}

At the landward and seaward boundary, salinity values are prescribed that are representative of fresh water, $0.5 \mathrm{psu}$, and sea water, $33.5 \mathrm{psu}$, respectively. A large horizontal diffusion coefficient is used in the landward and seaward zones (see Fig. 1) such that they contain entirely fresh or sea water during a computation. To find an along-channel residual density gradient in the cross-section of interest that is approximately constant, as assumed in the idealized model, a horizontal diffusion coefficient of $10 \mathrm{~m}^{2} s^{-1}$ is used in the intermediate zone.

\subsubsection{Numerical model grid}

The domain is discretized by using 480x 21 grid cells in the along-channel and lateral direction, respectively, with constant longitudinal $(\Delta x=2.5 \mathrm{~km})$ and cross-channel $(\Delta y=250 \mathrm{~m})$ grid size. The water column is represented by ten layers, with thickness increasing gradually from $2 \%$ of the water depth for the lowest (i.e. near-bed) layer to $20 \%$ for the top layer.

\subsection{Idealized model setup}

The idealized model uses the same Gaussian crosschannel bed profile as the numerical model at $x=$
$800 \mathrm{~km}$. The semi-diurnal tidal discharge and residual density gradient are derived from the numerical model results by means of harmonic analysis and used as input for the idealized model.

\subsection{Comparing the results}

The outcome of both models will be represented by harmonic constituents of quantities (residual, semidiurnal part etc.) rather than their temporal behavior. We will denote tidal constituents by subscripts, e.g., $u_{\mathrm{M} 2}$ is the semi-diurnal part of the along-channel velocity.

The numerical and idealized model results will be compared quantitatively by comparing figures, but also qualitatively by using a correlation function. The correlation function is an adequate tool to quantify the qualitative agreement of quantities. It can be obtained as follows. Let $p(y, z)$ and $P(y, z)$ denote a spectral component of a quantity obtained by the numerical and idealized model, respectively. Let their cross-sectional averages be denoted by $\bar{P}$ and $\bar{p}$. Also define the functional $[P, p]$ as the cross-sectional integral

$$
[P, p]=\int_{0}^{\mathrm{B}} \int_{-\mathrm{H}(\mathrm{y})}^{\zeta} P(y, z) p(y, z) \mathrm{d} z \mathrm{~d} y .
$$

The correlation coefficient $r(P, p)$ between the fields $\mathrm{P}$ and $\mathrm{p}$ is then defined as

$$
r(P, p)=\frac{[P-\bar{P}, p-\bar{p}]}{\sqrt{[P-\bar{P}, P-\bar{P}][p-\bar{p}, p-\bar{p}]}} .
$$

By definition, $r(P, p)$ varies between -1 and 1 .

\section{RESULTS}

\subsection{Default case}

The parameter settings for this case are listed in Table 1 . The tidal discharge velocity $U$ is defined as the semi-diurnal discharge $Q$ divided by the crosssectional area below the mean water level. At the seaward side, a vertical $M_{2}$ tide (water level $\zeta$ ) with an amplitude of $1.5 \mathrm{~m}$ is imposed. At the landward opening, the discharge is zero.

\subsubsection{Validity of model assumptions}

The numerical model setup described in Sect. 3 is able to reproduce the most important physical conditions of the idealized model. In particular, the assumption of along-channel uniform conditions and the rigid lid approximation are obeyed to good approximation.

The residual density variation from the numerical model is used to obtain the residual across-channel density gradient that drives the baroclinic flows in the idealized model. The along-channel component is approximately constant and is $1.03 \mathrm{kgm}^{-4}$. The residual 
General parameter settings

\begin{tabular}{|c|c|l|}
\hline \hline Symbol & Meaning & Value \\
\hline$H_{\mathrm{e}}$ & Entrance depth & $15 \mathrm{~m}$ \\
$A_{\mathrm{v}}, K_{\mathrm{v}}$ & Vertical viscosity/diffusion & $0.01 \mathrm{~m}^{2} \mathrm{~s}^{-1}$ \\
$K_{\mathrm{h}}$ & Horizontal diffusion & $10 \mathrm{~m}^{2} \mathrm{~s}^{-1}$ \\
$H_{\max }$ & Maximum depth & $30 \mathrm{~m}$ \\
$H_{\min }$ & Minimum depth & $2 \mathrm{~m}$ \\
\hline
\end{tabular}

Output from TRIWAQ / input for idealized model

\begin{tabular}{|c|c|l|}
\hline \hline Symbol & Meaning & Value \\
\hline$Q$ & $M_{2}$ discharge amplitude & $1.5 \times 10^{4} \mathrm{~m}^{3} \mathrm{~s}^{-1}$ \\
$U$ & $M_{2}$ discharge velocity & $0.19 \mathrm{~ms}^{-1}$ \\
$\frac{\partial \rho}{\partial x}$ & Parallel density gradient & $\sim 10^{-4} \mathrm{kgm}^{-4}$ \\
$\frac{\partial \rho}{\partial y}$ & Lateral density gradient & $\sim 10^{-4} \mathrm{kgm}^{-4}$ \\
\hline
\end{tabular}

Table 1: Parameter values for the default case.

across-channel gradient shows only mild vertical variation ( $\sim 20 \%$ of the vertical mean). Consequently, the lateral variation of $\partial\langle\rho\rangle / \partial y$ is well represented by its vertically averaged profile and ranges from $-5 \times 10^{-5} \mathrm{kgm}^{-5}$ to $1.5 \times 10^{-4} \mathrm{kgm}^{-4}$ (see Fig. 2). Hence the main assumptions of the idealized model regarding the residual horizontal density gradient are satisfied.

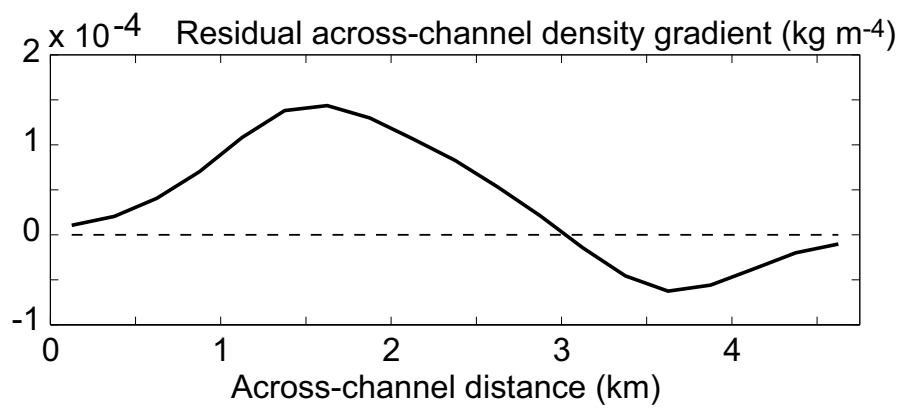

Figure 2: Lateral gradient of the residual density that drives the lateral residual flow in the idealized model.

\subsubsection{Residual and semi-diurnal flow patterns}

Figure 3 compares the cross-sectional variation of the along-channel directed residual flow. This result shows a landward directed flow in the deep parts while a seaward return current is located more towards the surface.

The qualitative agreement between the numerical and idealized model is good. The maximum velocities are similar in both models, and range from $-7 \mathrm{~cm} \mathrm{~s}^{-1}$ to $8 \mathrm{~cm} \mathrm{~s}^{-1}$. One remarkable difference, though, is that the landward directed flow in the idealized model extends throughout the whole water column in the deepest part of the channel, while this is not the case for the TRIWAQ result.

In Fig. 4, the residual lateral flow is plotted. For both models, we find a double gyre pattern which is
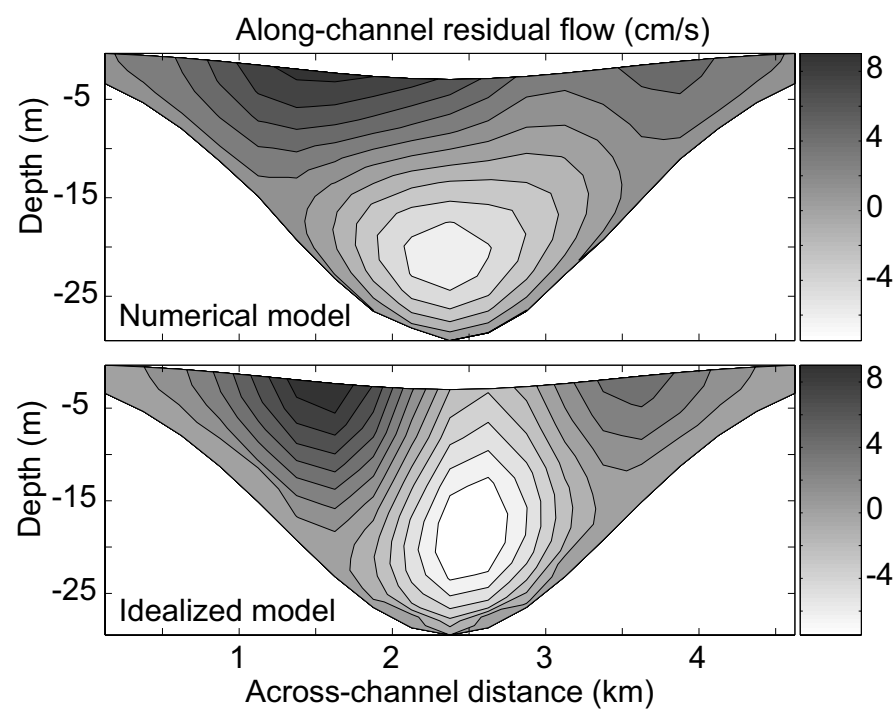

Figure 3: Along-channel directed residual flow from numerical (top panel) and idealized model (bottom). Positive (negative) values refer to down-estuary (upestuary) flow. Orientation of this and subsequent figures is looking up-estuary.The depression near the surface is an artefact of the harmonic analysis, which cannot be performed on the entire uppermost sigmalayer since it is intertidal. The correlation between the two results is 0.85 .

diverging near the bed and converging near the surface. The double gyre is clearly asymmetric, with the left part showing stronger flow velocities than the right one. The typical velocities are comparable in both models and vary between $-2 \mathrm{~cm} \mathrm{~s}^{-1}$ and $3 \mathrm{~cm} \mathrm{~s}^{-1}$, although the idealized model tends to predict somewhat higher velocities.

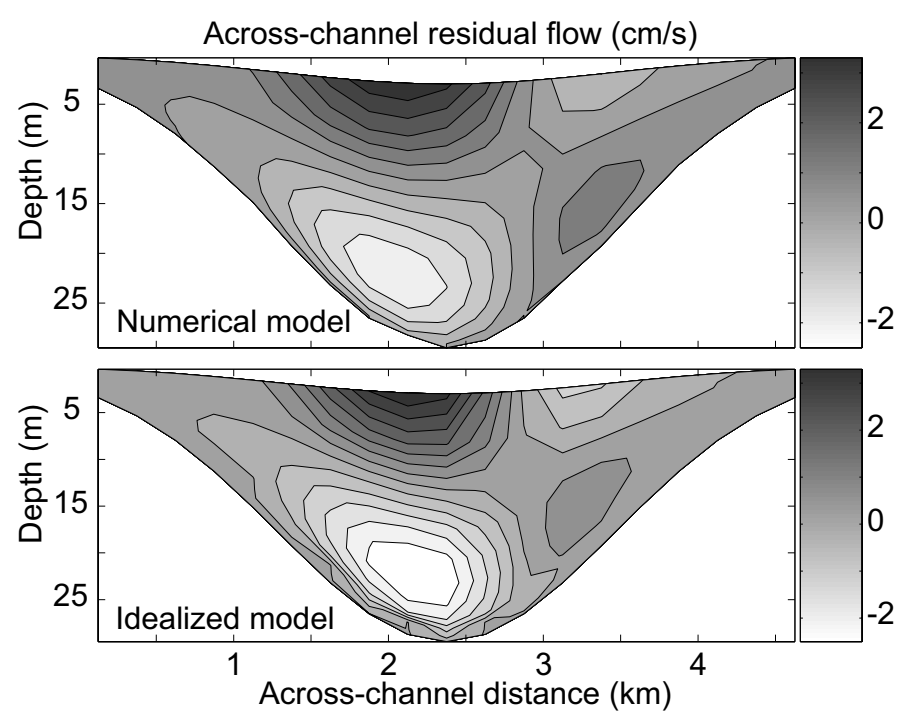

Figure 4: Lateral component of the residual flow from numerical (top panel) and idealized model (bottom). The correlations for the lateral and vertical velocity are 0.98 and 0.97 , respectively.

The results for the semi-diurnal part of the alongchannel velocity are compared in Fig. 5 and Fig. 6. 
The former plot shows the instantaneous flow at maximum flood (i.e. when the tidal discharge $Q$ is minimum) while the latter gives the flow at slack following the flood (i.e. when $Q=0$ ). These results show a good qualitative and quantitative agreement. The correlation coefficients for amplitude and phase distribution are 0.99 and 0.81 , respectively.

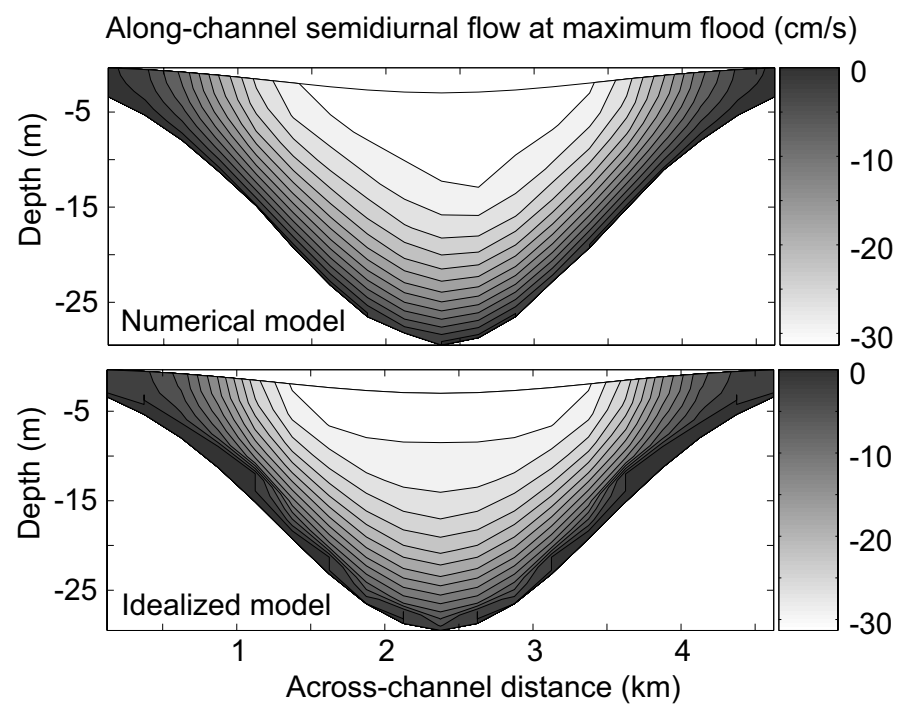

Figure 5: Distribution of the instantaneous longitudinal component of the semi-diurnal cross-sectional flow at maximum flood. The top panel refers to the TRIWAQ result while the bottom plot shows the idealized model result.

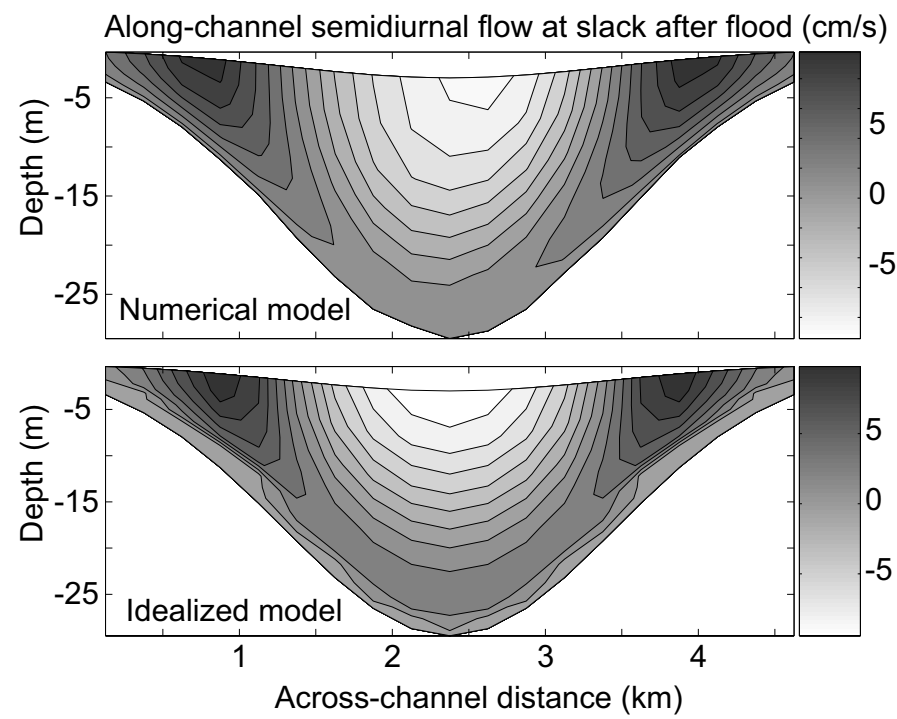

Figure 6: Picture of the semi-diurnal longitudinal flow at slack following flood. The top panel is the result from the numerical idealized model while the bottom plot refers to the idealized model.

The semi-diurnal component of the cross-sectional water motion consists of a single cell circulation pattern (Fig. 7 and 8). The corresponding lateral velocity $\left(v_{\mathrm{M} 2}\right)$ is compared in Fig. 7, which shows the semidiurnal flow at maximum flood. In Fig. 8, the situa- tion at slack after flood is displayed. In both pictures, the schematic arrows indicate the instantaneous transverse circulation. The lateral flow velocities reach val-

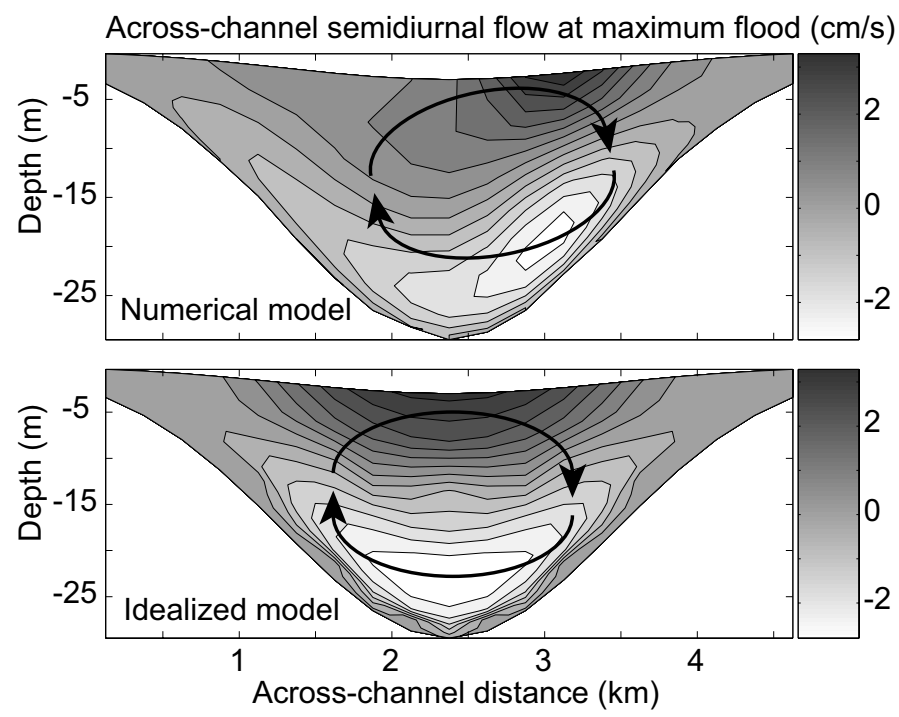

Figure 7: Lateral component of the semi-diurnal cross-sectional flow at maximum flood. The top panel shows the result from TRIWAQ while the bottom figure refers to the idealized model.

ues up to $\sim 4 \mathrm{~cm} \mathrm{~s}^{-1}$ and are similar for both models. The numerical model, however, reveals a strongly asymmetric double cell circulation at slack (Fig. 8) while the idealized model gives a single gyre. The correlation coefficients for amplitude and phase distribution of the lateral velocity component are 0.93 and 0.40 , respectively.

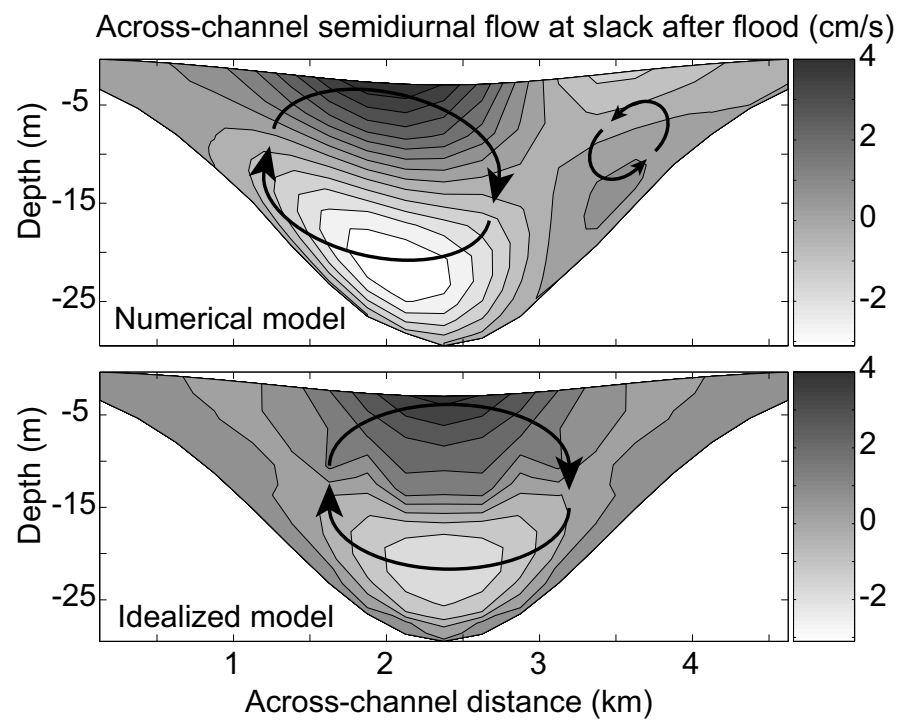

Figure 8: The semi-diurnal lateral flow at slack following maximum flood. The top and bottom panels are obtained from the numerical and idealized model, respectively. The instantaneous circulation is indicated by arrows. 


\subsubsection{Sediment}

Figure 9 compares the mean sediment concentration from both models as well as the sediment availability at the bed. From this plot we see that both the flow
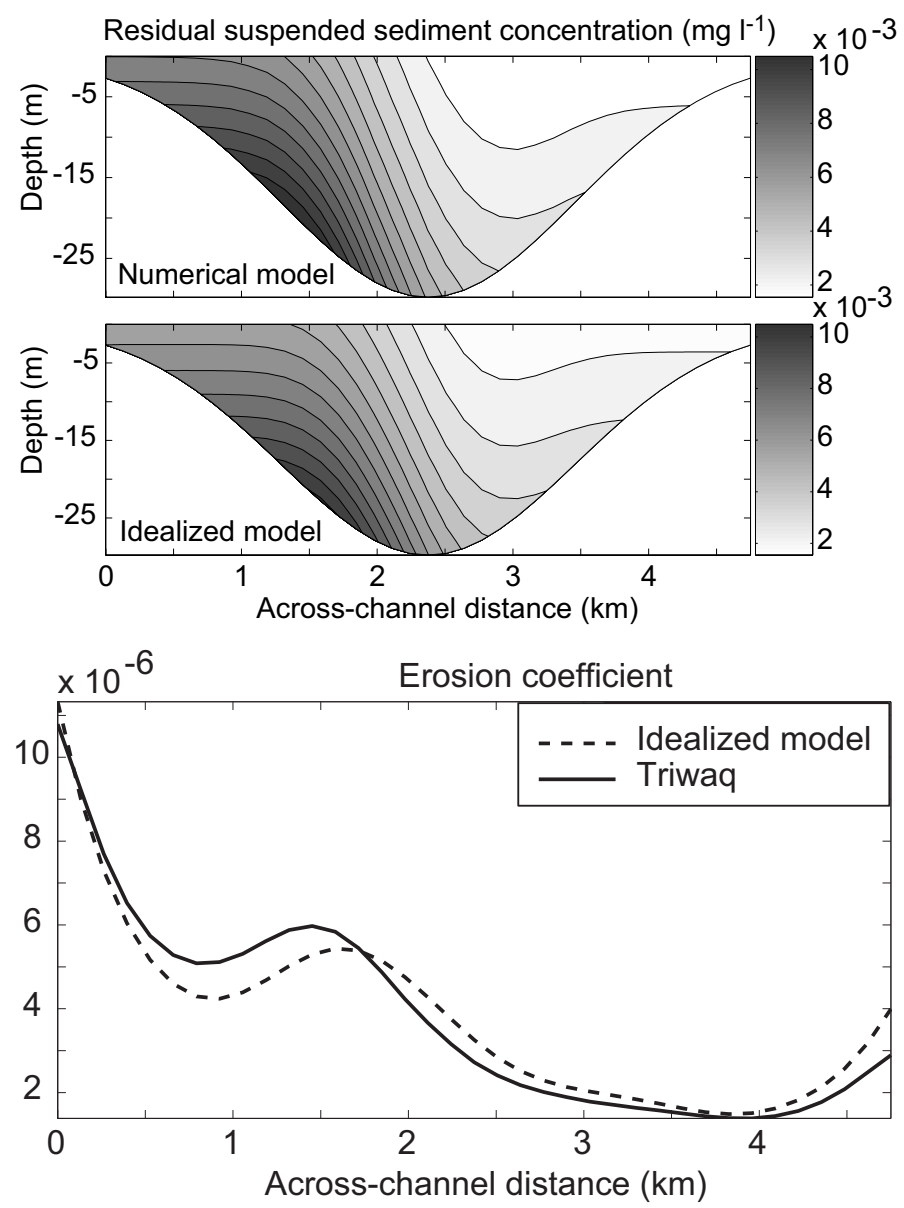

Figure 9: Tidally-averaged sediment distribution (in $\mathrm{mg}^{-1}$ ) from the numerical model (top panel) and the idealized model (middle panel). The correlation is 0.98 . The bottom plot shows a comparison of the sediment availability at the bed.

from the numerical and idealized model give a mean sediment distribution that is concentrated on the left side of the channel cross section. Also, the values of the sediment concentrations agree well. Likewise, the availability of sediment is similar for both models.

\subsection{Sensitivity to tidal flow conditions}

Here, we will compare model results that have different tidal flow conditions than the default case. The tidal discharge velocity $U$ is varied from 0.02 to $0.55 \mathrm{~m} \mathrm{~s}^{-1}$, which was achieved by varying the entrance depth $H_{\mathrm{e}}$ from 8 to $30 \mathrm{~m}$.

\subsubsection{Flow patterns}

The results for the flow comparison are summarized in Fig. 10 which shows the correlations for the residual and semi-diurnal parts of the velocity components as a function of U. From these plots, we can draw sev-

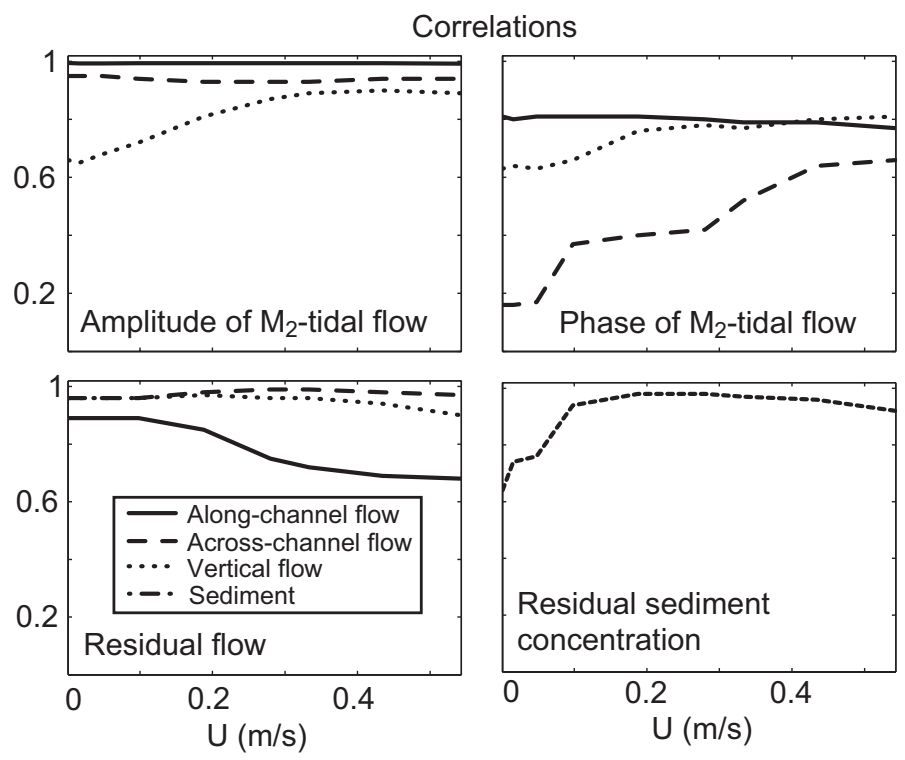

Figure 10: Variation of the correlation for residual and semi-diurnal flow as well as the residual sediment concentration.

eral conclusions. First, we see that the amplitude and phase of the along-channel semi-diurnal tide $\left(u_{\mathrm{M} 2}\right)$ agree well in both models. The qualitative behavior of the cross-channel tidal velocities $\left(v_{\mathrm{M} 2}\right.$ and $\left.w_{\mathrm{M} 2}\right)$ is also rather similar, although the correlations are poor for low tidal discharge velocity. This holds especially for the phases.

Regarding the residual flows, there is an overall good agreement between the idealized and numerical model results. The only noticeable deviation is observed for the along-channel residual flow at higher tidal flow conditions, where the correlation drops to below 0.7 . To elaborate on this, we have plotted $u_{\mathrm{M} 0}$ at $U=0.54 \mathrm{~m} \mathrm{~s}^{-1}$ for both models in Fig. 11. We see, that the idealized model gives a residual flow that is up-estuary (down-estuary) on the right (left) bank while the numerical model result shows up-estuary flow in the deeper parts and down-estuary velocities near the surface.

\subsubsection{Mean sediment distribution}

The bottom right panel in Fig 10 shows the correlation for the mean lateral sediment distribution. We see that model results in general agree rather well, except for low tidal flow velocities.

\section{DISCUSSION}

\subsection{The default case}

In Sect. 4.1 we have presented a case for which the idealized and numerical model results agree well for both the water motion and lateral sediment distribution. This indicates that the idealized model gives a proper description of the relevant physical mechanisms that govern the cross-channel distribution of flow and sediment. 


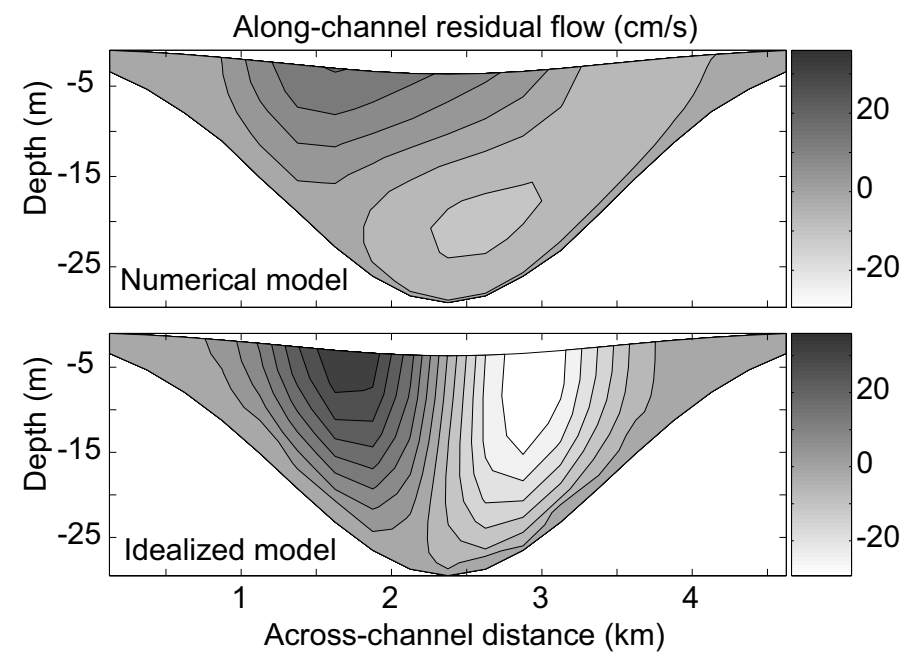

Figure 11: Comparison of along-channel residual flow at $U=0.54 \mathrm{~m} \mathrm{~s}^{-1}$. The correlation is 0.68

\subsection{Sensitivity of model results to tidal conditions}

The results presented in Section 4.2 showed that the residual and semi-diurnal flow patterns are similar for a fairly wide range of tidal flow conditions. Some differences were found as well.

\subsubsection{Semi-diurnal flow}

Regarding the semi-diurnal flow, we have found a good match for the along-channel velocity component. This suggests that the main longitudinal force balance in the idealized model (gravity wave balance versus friction) also governs the along-channel dynamics in the numerical model. The semi-diurnal part of the transverse flow $\left(v_{\mathrm{M} 2}\right.$ and $\left.w_{\mathrm{M} 2}\right)$ is mostly generated by Coriolis deflection of the along-channel tide. The similarity of model results for these velocity components is less at low tidal discharge velocity. This discrepancy might be related to the semi-diurnal across-channel density gradient, which is not included in the idealized model.

\subsubsection{Residual flow}

The residual velocities also compare well. The only qualitative deviations are found for the longitudinal flow at high tidal flow conditions (see, e.g., Fig. 11). At low tidal flow conditions, the along-channel residual flow consists mainly of a classical gravitational circulation (inflow in deeper parts, outflow near surface as can be seen in Fig. 3)). At high tidal flow conditions, the idealized model flow is dominated by a nonlinear contribution resulting from tidal advection of along-channel momentum (inflow at the right, outflow at the left as can be seen in the bottom panel of Fig. 11). In the corresponding Triwaq flow, the contribution by tidal advection is also present. However, its contribution is less strong, leading to distortion of the classical gravitational flow that dominates for low $U$ (compare numerical model results in Fig. 3 and 11).
The residual transverse flow is very similar in both models. In the idealized model, a net lateral circulation is predominantly generated by a prescribed residual cross-channel density gradient. This gradient is taken from the numerical model result. Apparently, the net cross-channel circulation is described qualitatively well, provided that the residual density gradient is appropriate.

\subsubsection{Mean sediment concentration}

The results presented in Sect. 4.2.2 show a rather good similarity between the model results. The main reason for this good match can be seen if one considers the sediment balance (Eq. 1) for the default case in more detail (Fig. 12). As for the other flow con-

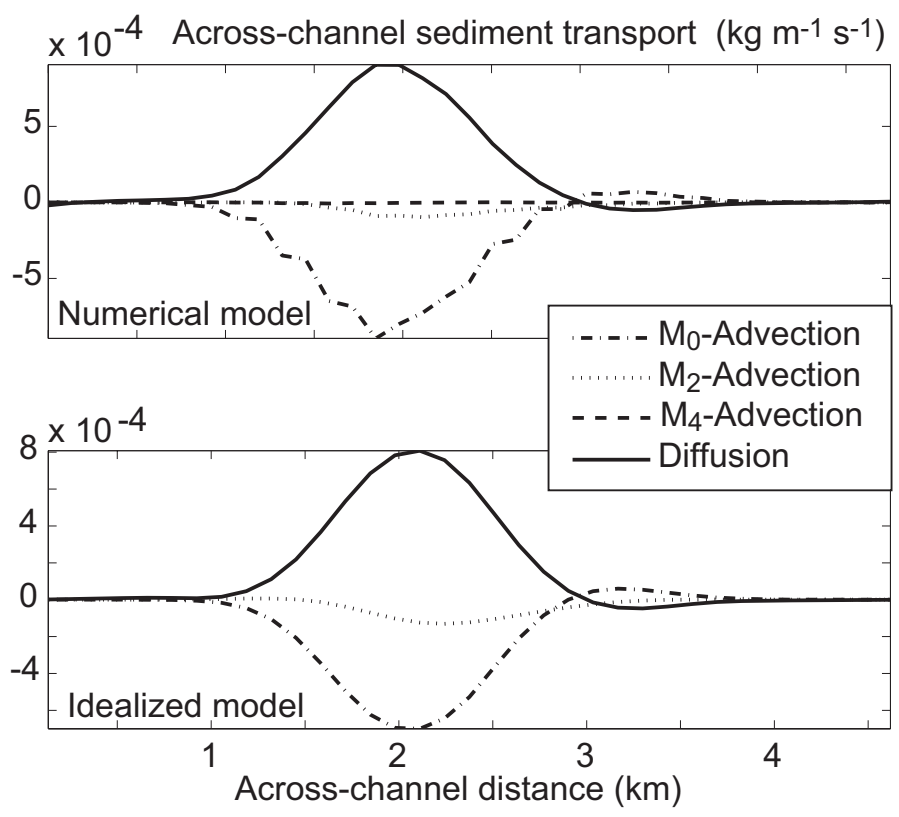

Figure 12: Contributions to the total sediment balance from the numerical model (top panel) end the idealized model (middle panel). The solid line denotes the diffusive sediment flux, while the other lines represent the contribution of individual harmonic components to the mean advective flux.

ditions, the diffusive sediment flux for both models is mainly balanced by the advective sediment flux resulting from advection of the residual sediment concentration by the residual across-channel flow ("M0advection" in Fig. 12). This sediment balance to good approximation involves only the mean lateral velocity $v_{\mathrm{M} 0}$ and the semi-diurnal longitudinal flow $u_{\mathrm{M} 2}$, which gives the dominant contribution to $c_{\mathrm{M} 0}$ through $\langle\tau\rangle$. Hence, differences between the model results regarding the lateral semi-diurnal ("M2-advection") water motion have only a limited effect. Similarly, the contribution of quarterly diurnal flow and sediment to the sediment balance ("M4-advection", included in the numerical model) appears to be insignificant.

Only for low tidal discharges, deviations between the 
models are found. This is a consequence of the fact that the residual bed shear stress is computed differently in both models as explained in Sect. 2.2. This causes a discrepancy in the computed residual sediment concentration.

\section{CONCLUSIONS AND FUTURE RESEARCH}

We have compared the outcome of an idealized and a numerical model as a means to gain insight regarding the lateral distribution of flow and sediment in tidal estuaries. Also, tidal conditions have been varied to probe the validity of the idealized model.

The general conclusion is, that the results presented in this contribution give a good qualitative agreement between both models. This indicates that, for most cases considered here, the idealized and numerical model grasp a good deal of the relevant physical mechanisms. Hence, the idealized model may be considered as a useful tool to analyze the results of the complex numerical model used in this paper.

The only major deviations between the models were found at either high or low tidal flow conditions. For strong tidal flows, the idealized model overestimates the effect of along-channel residual flow generated by advective effects. However, contrary to the numerical model, the idealized model is able to identify this advective contribution in isolation. It can then be used to predict an increase of its importance of the advective effects as the tidal flow $U$ becomes stronger. This trend can be used to interpret the numerical model results (cf. Fig. 3 and 11).

For low tidal flows, the cross-channel semi-diurnal circulations show poor correlation, presumably because the semi-diurnal cross-channel density gradient is not included in the idealized model. The residual flows, however, do match despite the fact that low values of $U$ violate the assumption that the hydrodynamics are tidally dominated. At this point, the idealized model is valid beyond its strict range of applicability. This can be explained as follows. The idealized model predicts that, if tides are weak, the residual flow is governed by the residual density gradients. Hence, in this case the idealized model is expected to yield reasonable results.

Regarding the lateral distribution of residual sediment one finds a good overall agreement, even if semi-diurnal flow patterns show considerable deviations. This is because the mean transverse sediment transport is mainly affected by the along-channel tidal flow, the residual across-channel flow and the mean sediment concentration. The time-averaged bed shear stress (which determines residual erosion, hence $\left.c_{\mathrm{M} 0}\right)$ is mostly determined by the (dominant) alongchannel semi-diurnal flow $\left(u_{\mathrm{M} 2}\right)$. The other flow components are an order of magnitude smaller so that they give a less important contribution to mean sediment transport. Only at low tidal flow conditions, the sediment distributions disagree. This is because the bed shear stress computation for the idealized model assumes a tidally dominated flow, which is not valid at low values for $U$.

The results presented here considered only a variation of tidal flow conditions at rather high values of eddy viscosity and diffusivity $\left(\sim 0.01 \mathrm{~m}^{2} \mathrm{~s}^{-1}\right)$. Hence, the vertical variation of density is mild so that the density gradient is well approximated by its vertical average. This is consistent with the assumptions of the idealized model. For lower values of vertical mixing, one may expect strong effects of density stratifications that are not included in the idealized model. A comparison of model results for this regime is still under consideration and will be presented elsewhere.

\section{ACKNOWLEDGEMENTS}

G.P. Schramkowski was funded by the Maritime Access Division of the Flemish authorities. TRIWAQ is part of the SIMONA modelling environment which is property of the Dutch Ministry of Transport, Public Works and Water Management (Rijkswaterstaat).

\section{REFERENCES}

Fugate, D. C., C. T. Friedrichs, and L. P. Sanford (2007). Lateral dynamics and associated transport of sediment in the upper reaches of a partially mixed estuary, Chesapeake Bay, USA. Cont. Shelf Res. 27, 679-698.

Geyer, W. R., R. P. Signell, and G. C. Kineke (1998). Lateral trapping of sediment in a partially mixed estuary. In J. Dronkers and M. B. A. M. Scheffers (Eds.), Physics of Estuaries and Coastal Seas, Brookfield, Vt., pp. 115124. A. A. Balkema.

Huijts, K. M. H., H. M. Schuttelaars, H. E. de Swart, and C. T. Friedrichs (2007). Transverse structure of 3d-residual flow in tidal estuaries - an analytical study. Submitted to J. Geophys. Res..

Huijts, K. M. H., H. M. Schuttelaars, H. E. de Swart, and A. Valle-Levinson (2006). Lateral trapping of sediment in tidal estuaries: an idealized model. J. Geophys. Res. 111, C12016, doi:10.1029/2006JC003615.

Nichols, M. M. (1972). Sediments of the James River estuary, Virginia. Mem. Geol. Soc. Am. 133, 169-210.

Stelling, G. S. (1984). On the construction of computational methods for shallow water flow problems. Comm. 35, Rijkswaterstaat. 